\title{
Heterogeneity of Cerebral Capillary Flow in Man and Its Consequences for Estimation of Blood-Brain Barrier Permeability
}

\author{
Marianne M. Hertz and Olaf B. Paulson, Departments of Psychiatry and \\ Neurology, Rigshospitalet, State University Hospital, Copenhagen, Denmark
}

\begin{abstract}
A B S T R A C T Blood-brain barrier permeability studies made in man using the indicator dilution method revealed that the extraction of the test substance increases during the upslope of the venous (outflow) dilution curve. The present study aimed to obviate the possibility that this could result from intravascular phenomena, such as interlaminar diffusion (the result of differences in molecular size) and erythrocyte carriage. Several reference substances were employed for the determination of the extraction in order that careful correction could be made for differences in intravascular behavior of the test and reference substance. The test substances studied were D-glucose, L-phenylalanine, water, propranolol, and benzodiazepines, representing both carrier-transported and lipophilic substances. In-diethylenetriamine pentaacetic acid, $\mathrm{Na}^{+}, \mathrm{Cl}^{-}, \mathrm{L}$-glucose, and $\mathrm{L}$-lysine were employed as reference substances.
\end{abstract}

For all the substances tested, and after correction for intravascular phenomena, the extractions were found to increase during the initial part of the dilution curve. This increasing extraction can be ascribed to heterogeneity of the cerebral circulation; the higher extraction corresponds to longer contact with the blood-brain barrier and indicates a longer transit time. Signs of heterogeneity were also present when blood flow was elevated above normal. Any influence that heterogeneity might have on the mean extraction value can be minimized by using an appropriate calculation of the extraction of the test substance.

\section{INTRODUCTION}

The indicator dilution method (1) has been widely used for the quantitative determination of capillary permeability and adapted for studies of the blood-brain barrier in man (2-4). We are using this method in a

Received for publication 5 April 1979 and in revised form 19 December 1979. number of ongoing ivestigations (such as the effect of insulin on the cerebral uptake of glucose) and we have observed some basic features of the dilution and extraction curves indicating heterogeneity of the cerebral microcirculation. These aspects will be the subject of this paper.

The method consists of a rapid injection into the internal carotid artery of test and reference substances, followed by collection of blood samples from the internal jugular vein for determination of the dilution curves and calculation of the extraction (loss to brain tissue) of the test substance.

Ideally, the test substance and the intravascular reference substance should have the same intravascular behavior with regard to diffusion and erythrocyte carriage. When that is the case, the fractional loss (the extraction $[E])^{1}$ of the test substance can be calculated in each venous sample. Generally, however, this ideal requirement cannot be met and corrections must be applied to compensate for differences in intravascular behavior.

The theory of the method is based on a single capillary model; the assumption is made that the capillary bed studied is homogenous. Accordingly, tracer particles with short transit times should pass capillaries with the same flow rates, lengths, and permeabilities as tracer particles with long transit times. With this assumption, it is only necessary to use the upslope of the outflow curves for calculation of the average $E$; the downslope, which is influenced by backdiffusion of test substance, can be disregarded.

In our investigations, we observed that the $\mathrm{E}$ of glucose increases with time, making estimation of the average $\mathrm{E}$ difficult (Fig. 1). With other test substances we found a similar increase of $E$ during the upslope

\footnotetext{
${ }^{1}$ Abbreviations used in this paper: CBF, cerebral blood flow; DTPA, diethylenetriamine pentaacetic acid; E, extraction; $t$, time.
} 


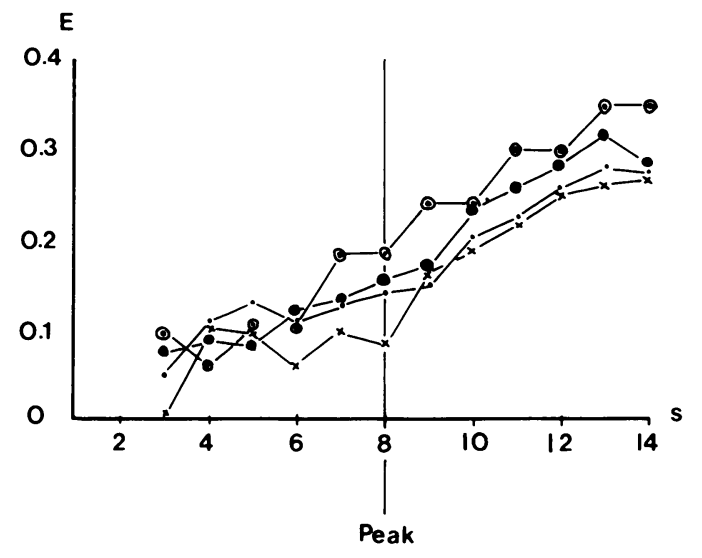

Figure 1 E curves for glucose from four different subjects illustrating the steep increase in $\mathrm{E}$ (and the difficulty in assessing a true average $\mathrm{E}$ ).

of the tracer dilution curves; this seems to indicate heterogeneity of the microcirculation in the human brain.

However, to obviate the possible influence of intravascular phenomena on the shape of the $E$ curve, it was necessary to apply corrections for the differences in intravascular behavior of the test and reference molecules. These corrections, which are detailed in this paper, show that the increase in $\mathrm{E}$ during the upslope of the dilution curve is not the result of intravascular phenomena, but is likely to be the result of capillary heterogeneity. The implications of these findings for the calculation of the average $\mathrm{E}$ are discussed.

\section{METHODS}

Informed consent having been obtained, 42 patients hospitalized for various cerebral disorders requiring carotid angiograms were studied in connection with the angiography. Patients with major cerebral lesions (e.g., larger tumors) that might interfere with blood-brain barrier function were excluded.

Using the Seldinger technique, percutaneous punctures in the neck made under local anaesthesia, a small polyethylene catheter (external diameter $1.7 \mathrm{~mm}$ ) was introduced into one of the internal jugular veins and a second catheter (external diameter $1.2 \mathrm{~mm}$ ) introduced into the internal carotid artery on the side appropriate for the angiogram. The tip of the venous catheter was placed in the superior bulb of the internal jugular vein; the tip of the arterial catheter was placed in the internal carotid artery just below the siphon. Correct positioning of the arterial catheter was verified by noting the absence of diffuse facial discoloration after a rapid injection of saline or Evans blue. At the end of the study, which usually lasted 20-30 min, the carotid catheter was used for the diagnostic angiogram.

For each determination of blood-brain barrier permeability using the indicator dilution method, a 2-4-ml bolus, containing a test compound and several intravascular reference compounds, was injected rapidly $(1-2 \mathrm{~s})$ through the indwelling intracarotid catheter. Starting a few seconds before the injection, a continuous series of $1.0-\mathrm{ml}$ blood samples was collected from the venous catheter by means of a sampling machine and then deposited into dry heparinized tubes at a speed of $1 \mathrm{sample} / \mathrm{s}$.

The test and reference substances contained in each injected bolus were: $10 \mu \mathrm{Ci}^{24} \mathrm{Na}^{+}, 40 \mu \mathrm{Ci}{ }^{113 m} \mathrm{In}$-diethylenetriamine pentaacetic acid (DTPA), $2 \mu \mathrm{Ci}^{36} \mathrm{Cl}^{-}, 40 \mu \mathrm{Ci}$ ${ }^{3} \mathrm{H}$-labeled substance (either $\mathrm{H}_{2} \mathrm{O}$, propranolol, L-phenylalanine, or D-glucose), and $10 \mu \mathrm{Ci}{ }^{14} \mathrm{C}$-labeled compound (benzodiazepine, L-glucose, or L-lysine). Thus, two $\gamma$-emitters $\left({ }^{24} \mathrm{Na}\right.$ and $\left.{ }^{113 \mathrm{~m}} \mathrm{In}\right)$ and three $\beta$-emitters $\left({ }^{36} \mathrm{Cl},{ }^{3} \mathrm{H}\right.$, and $\left.{ }^{14} \mathrm{C}\right)$ were contained in each bolus. ${ }^{24} \mathrm{Na}^{+}$and ${ }^{3} \mathrm{HOH}$ (labeled water) were obtained from Ris $\phi$, Denmark, DTPA from CIS, International CIS, Quintin, France, the benzodiazepines from Roche Diagnostics Div., Hoffman-La Roche Inc., Nutley, N. J., and the other isotopes from Amersham Corp., Arlington Heights, Ill. or NEN Chemicals, West Germany.

A standard solution was prepared from each bolus by adding an aliquot of injectate to venous blood sampled in a dry heparinized tube before the injection. When repeated studies of the blood-brain barrier were performed, a time interval of 10 min was allowed between measurements.

All isotopes were counted in the plasma of the blood samples, the $\gamma$ - and $\beta$-emitters being counted in the same sample. ${ }^{24} \mathrm{Na}^{+}$and ${ }^{113 \mathrm{~m}} \mathrm{In}$ were counted in a crystal scintillation counter (Packard autogamma 5385, Packard Instrument Co., Inc., Downers Grove, Ill.), appropriate corrections for channel spillover and decay applied. After decay of the $\gamma$-emitters, ${ }^{36} \mathrm{Cl},{ }^{3} \mathrm{H}$, and ${ }^{14} \mathrm{C}$ were assayed using liquid scintillation counting (scintillation fluid: Instagel, spectrometer; Packard Tricarb 3375) and corrections for quenching and channel spillover were applied using the method of external standardization.

The cerebral blood flow (CBF) was measured during each study using the ${ }^{133} \mathrm{Xe}$ intra-arterial injection method; 3-4 $\mathrm{mCi}$ of ${ }^{133} \mathrm{Xe}$ dissolved in $2-3 \mathrm{ml}$ of isotonic saline were injected rapidly $(1-2 s)$ into the internal carotid artery through the indwelling catheter. The clearance of the isotope from the hemisphere was followed by an externally positioned array of 16 small scintillation detectors; the average hemispheric blood flow was calculated from the initial slope of the semilogarithmically recorded clearance curves (5). Using a conventional electrode, the arterial carbon dioxide tension was determined concomitant with each $\mathrm{CBF}$ measurement.

Calculations. At any time $(t)$ of the tracer dilution curve, the $E$ of the test substance can be calculated as:

$$
E(t)=\frac{C_{\text {ref }}(t)-C_{\text {test }}(t)}{C_{\text {ref }}(t)},
$$

where $\mathrm{C}_{\text {rer }}(\mathrm{t})$ and $\mathrm{C}_{\text {test }}(\mathrm{t})$ are the relative concentrations of the reference and test substance (i.e., counting rate in blood sample divided by counting rate in the standard) corresponding to $t$.

$\mathrm{E}(\mathrm{t})$ would represent the true unidirectional fractional transcapillary escape of the test molecule if no intravascular separation of test and reference substance occurred and if only insignificant backdiffusion took place. Backdiffusion is more pronounced if $E$ is high, especially during the later part of the venous outflow curves. In the present study, no corrections for backdiffusion were applied because we were mainly interested in the shape of the first part of the $E(t)$ curve. Furthermore, backdiffusion would result in a decrease of $E(t)$ with $t$, whereas we observed the opposite.

Intravascular separation (interlaminar diffusion and erythrocyte carriage) of the test and reference substances is an important phenomenon that influences the shape of the $E(t)$ curves $(2-4,6)$; this in turn influences the calculation of the average $E$ considerably. In previous publications we mini- 
mized the influence of intravascular separation by using test and reference substances with closely matching intravascular properties and then calculating the average $E$ by integrating a region of the outflow curves appropriate to test-reference substances used. To obtain a more accurate reference curve for each test substance, additional corrections for intravascular separation have now been applied. This involves the simultaneous use of multiple intravascular reference substances and permits correction for both interlaminar diffusion and erythrocyte carriage. The principles of interlaminar diffusion, erythrocyte transport, and the corrections used for a more precise analysis of $E(t)$ curves will be described below.

Interlaminar diffusion (7) is the result of differences in the diffusibility of the test and reference substance, and results in two crossover points on the dilution curves and a $\mathrm{U}$-shaped $\mathrm{E}(\mathrm{t})$ curve (8) (Fig. 2). ${ }^{24} \mathrm{Na}^{+}$diffuses more rapidly than ${ }^{113 \mathrm{~m} I n-D T P A}$ away from the fast-moving axial part of the blood stream into the more slow-moving peripheral parts. Thus, on the upslope, the ${ }^{24} \mathrm{Na}^{+}$curve lies below that of ${ }^{113 \mathrm{~m} I n-D T P A}$, and $E(t)$ is positive. On the first part of the downslope the ${ }^{24} \mathrm{Na}^{+}$curve lies above that of ${ }^{113 \mathrm{~m}} \mathrm{In}-\mathrm{DTPA}$, and $E(t)$ is negative because diffusion of labeled sodium is now reversed, i.e., away from the slow-moving peripheral part of the blood stream into the fast-moving axial part. Later, when the slow-moving peripheral part of the blood stream reaches the venous outflow, ${ }^{24} \mathrm{Na}^{+}$has been washed out with the axial blood stream, the ${ }^{24} \mathrm{Na}^{+}$curve again lies below that of ${ }^{113 m} \mathrm{In}-\mathrm{DTPA}$, and $\mathrm{E}(\mathrm{t})$ is positive. When comparing albumin $\left(50,000 M_{\mathrm{r}}\right)$ and $\mathrm{Na}^{+}\left(24 M_{\mathrm{r}}\right)$, Taylor diffusion is constantly observed $(5,8)$. The difference in diffusibility between In-DTPA

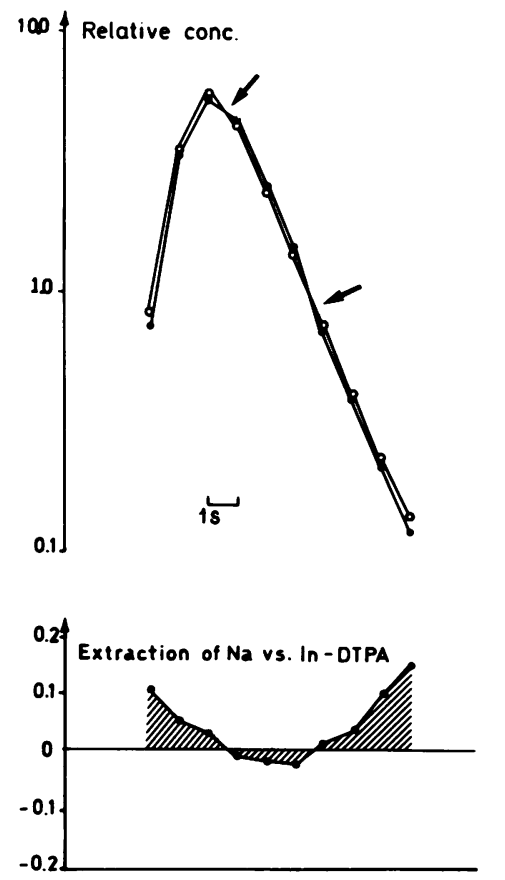

Figure 2 Intravascular interlaminar diffusion (Taylor phenomenon) is illustrated by two venous outflow curves and by the U-shape of the $\mathrm{E}$ curve. The crossover points are indicated by arrows. Note that the dilution curve for the smaller molecule $\mathrm{Na}^{+}$lies below that of In-DTPA during the upslope of the curves. $O$, In-DTPA;, $\mathrm{Na}$. $\left(506 M_{\mathrm{r}}\right)$ and $\mathrm{Na}^{+}$is, however, much smaller, and interlaminar diffusion is both less pronounced and less evident (in 28 of 41 investigations in the present study, two distinct crossovers between In-DTPA and $\mathrm{Na}^{+}$curves were observed).

To correct for interlaminar diffusion we used the ${ }^{113 \mathrm{~m}} \mathrm{In}$ - DTPA $-{ }^{24} \mathrm{Na}$ difference, which in the case of glucose was multiplied by 0.5 because the molecular weight of glucose (180) is halfway between that of $\mathrm{Na}^{+}$and In-DTPA.

Erythrocyte carriage (precession). A tracer that penetrates into the erythrocytes has a shorter transit time than a pure plasma tracer because the transit time of erythrocytes through the cerebral vascular bed is shorter than that of plasma (9). This is illustrated by comparing ${ }^{36} \mathrm{Cl}^{-}$with ${ }^{24} \mathrm{Na}^{+}$, both of which were used as intravascular reference substances in the present study. ${ }^{36} \mathrm{Cl}^{-}$penetrates the erythrocytes extremely rapidly $\left(t_{1 / 2} \sim 30 \mathrm{~ms}\right)(10)$, whereas ${ }^{24} \mathrm{Na}^{+}$essentially remains in plasma $\left(t_{1 / 2}\right.$ several hours). The molecular weights are 36 and 24 , respectively, and Taylor diffusion is thus negligible. The outflow curves in Fig. 3 demonstrate that the ${ }^{36} \mathrm{Cl}^{-}$curve lies above that of ${ }^{24} \mathrm{Na}^{+}$on the upslope, resulting in a negative $E(t)$. The curves then cross near the peak so that during the downslope the ${ }^{36} \mathrm{Cl}^{-}$curve is below that of ${ }^{24} \mathrm{Na}^{+}$, and $\mathrm{E}(\mathrm{t})$ is positive. This curve configuration is invariably present and was found in 38 of 41 investigations.

The difference between the ${ }^{36} \mathrm{Cl}^{-}$and ${ }^{24} \mathrm{Na}^{+}$curves was used to correct for erythrocyte transport. However, the equilibrium distribution between plasma and erythrocytes of the various tracers has to be taken into consideration. $\mathrm{For}^{36} \mathrm{Cl}^{-}$, the Gibbs-Donnan effect will maintain a concentration $30 \%$ lower

\section{VENOUS OUTFLOW CURVES}

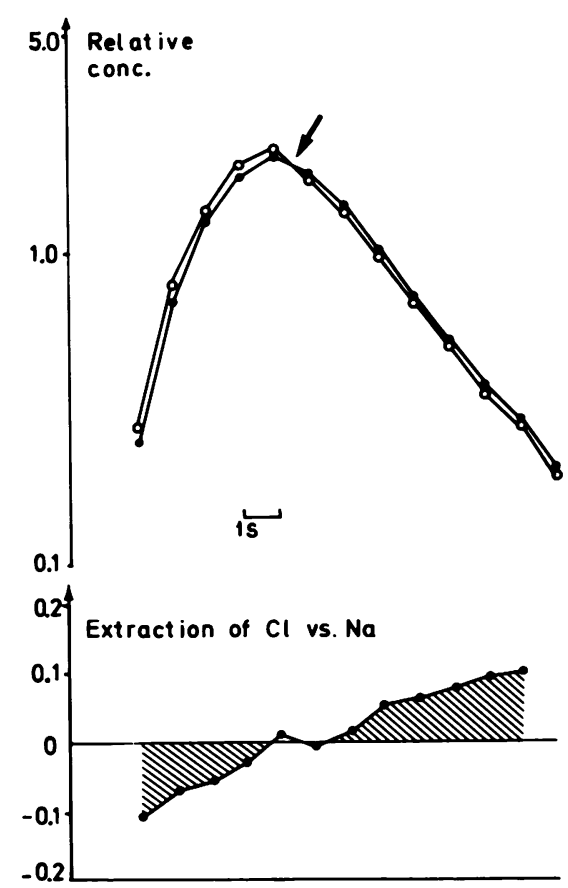

Figure 3 Erythrocyte carriage is illustrated by the one crossover point on the downslope of the venous outflow curve and by the rising $E$ curve. Note that the venous outflow curve of $\mathrm{Cl}^{-}$(which enters the erythrocytes) lies above that of $\mathrm{Na}^{+}$during the upslope of the curve. $\mathrm{O}, \mathrm{Cl} ;$ 
in the erythrocyte water phase than in the plasma water phase (11). Thus, in whole blood (at normal hematocrit), $25 \%$ of ${ }^{36} \mathrm{Cl}^{-}$ will be in the erythrocytes. In contrast, D-glucose distributes equally in plasma and erythrocyte water phases, $\sim 35 \%$ being carried in the erythrocytes, i.e., $50 \%$ more than for chloride. Thus, for glucose, the ${ }^{36} \mathrm{Cl}^{-}-{ }^{24} \mathrm{Na}^{+}$difference multiplied by 0.5 was added to the ${ }^{36} \mathrm{Cl}^{-}$reference curve to correct for the surplus erythrocyte carriage of glucose.

Corrections of reference curves. The corrections were based on the simultaneous determination of dilution curves for all the test and reference substances involved. In the illustrations (Figs. 4 and 5), the noncorrected as well as corrected reference curves are given, and it is apparent that corrections are small.

$\mathrm{HOH}$ has a molecular size corresponding to that of $\mathrm{Cl}^{-}$ (no Taylor correction) and distributes equally in plasma and erythrocyte water so that the carriage of $\mathrm{HOH}$ is $50 \%$ more than that of $\mathrm{Cl}^{-}$. Thus, by adding $50 \%$ of $\mathrm{Cl}^{-}-\mathrm{Na}^{+}$difference to the $\mathrm{Cl}^{-}$curve, $\mathrm{Cl}^{-}$was used as the reference after correcting for the difference in erythrocyte carriage. Corrected $\mathrm{Cl}^{-}=\mathrm{Cl}^{-}+1 / 2\left(\mathrm{Cl}^{-}-\mathrm{Na}^{+}\right)$.

For D-glucose, two different reference substances were used: $\mathrm{Cl}^{-}$and $\mathrm{L}$-glucose. Relative to the transit time, it was assumed that D-glucose penetrates erythrocytes very quickly through the cerebral circulation and, therefore, erythrocyte carriage does not interfere with the estimation of blood-brain barrier permeability, a problem discussed in detail by Perl (12) and Goresky et al. (13). No relevant data on the permeability of human erythrocyte to glucose are available but the following data confirm the validity of the assumption. Double determinations of mean transit $t$ for glucose and $\mathrm{Cl}^{-}$were made in five patients, both with an injectate of the isotopes in saline and then with the isotopes in a preequilibrated erythrocyte bolus.

\section{VENOUS OUTFLOW CURVES}
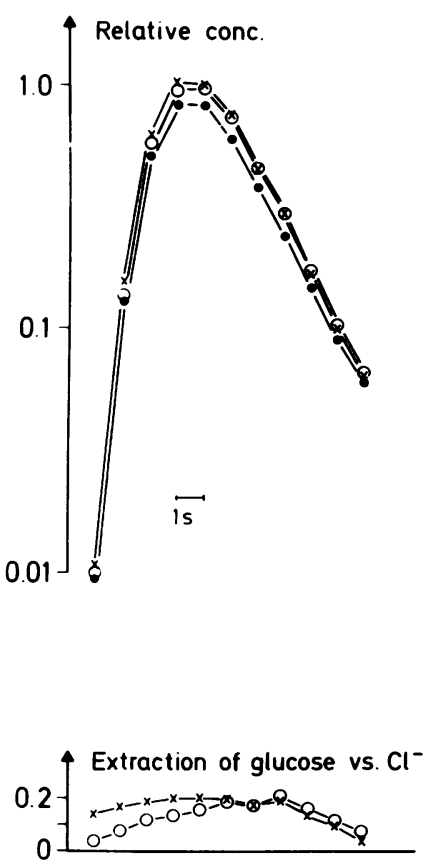

FIgURE 4 The increasing $\mathrm{E}$ of D-glucose during the upslope of the tracer dilution curve is illustrated. $\mathrm{O}, \mathrm{Cl}^{-}$, measured; $\times, \mathrm{Cl}^{-}$, corrected; $\boldsymbol{\bullet}$, Glucose.
No significant differences in $t$ were observed: with the saline bolus, $t$ for glucose was $0.07 \mathrm{~s}$ (mean) quicker than that for $\mathrm{Cl}^{-}$; with the preequilibrated erythrocyte bolus, glucose $\mathrm{t}$ was $0.04 \mathrm{~s}$ (mean) slower. In comparison, the difference between mean transit $t$ for erythrocytes and plasma is $1.1 \mathrm{~s}$ (9). The curve configuration and glucose $\mathrm{E}$ were also similar in the two situations.

(a) $\mathrm{Cl}^{-}$used as reference, corrected for difference in Taylor diffusion and difference in precession: the molecular size of D-glucose is about halfway between $\mathrm{Na}^{+}$and In-DTPA. The In-DTPA - $\mathrm{Na}^{+}$difference multiplied by 0.5 was used to correct for Taylor diffusion vs. $\mathrm{Cl}^{-}$. The correction for difference in precession is analogous to that used for $\mathrm{HOH}$. Corrected $\mathrm{Cl}^{-}=\mathrm{Cl}^{-}+1 / 2\left(\mathrm{In}-\mathrm{DTPA}-\mathrm{Na}^{+}\right)+1 / 2\left(\mathrm{Cl}^{-}-\mathrm{Na}^{+}\right)$.

(b) L-glucose used as reference, corrected for total difference in precession: L-glucose remains in plasma and there is no erythrocyte carriage, in contrast to $25 \%$ for $\mathrm{Cl}^{-}$and $35 \%$ for D-glucose. Thus, when L-glucose was used as the reference substance, the $\mathrm{Cl}^{-}$carriage multiplied by 1.5 was added to L-glucose to correct for the lack of erythrocyte carriage. Because molecular sizes are the same for L- and D-glucose, no correction for the Taylor effect was required. Corrected L-glucose $=$ L-glucose $+11 \frac{1}{2}\left(\mathrm{Cl}^{-}-\mathrm{Na}^{+}\right)$.

L-phenylalanine was compared with the reference substance L-lysine. Corrections were unnecessary because they have similar molecular size; both remain in the plasma during transit through the cerebral vascular bed.

Benzodiazepines and propranolol (molecular weights close to 300 ) have a high cerebral uptake, are lipid soluble, and,

\section{VENOUS OUTFLOW CURVES}
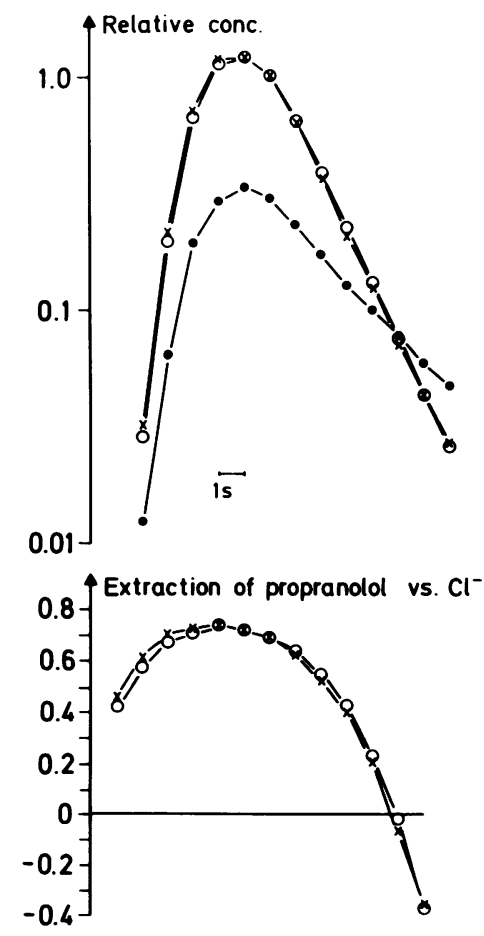

Figure 5 The increasing $\mathrm{E}$ of propranolol during the upslope of the tracer dilution curve is illustrated. Note that the correction for the intravascular phenomena is only minor and does not influence the $\mathrm{E}$ curve qualitatively. $\mathrm{O}, \mathrm{Cl}^{-}$, measured; $\times, \mathrm{Cl}^{-}$, corrected; $\bullet$, Glucose. 
TABLE I

Variations in Extractions Corresponding to Various Points on the Dilution Curves

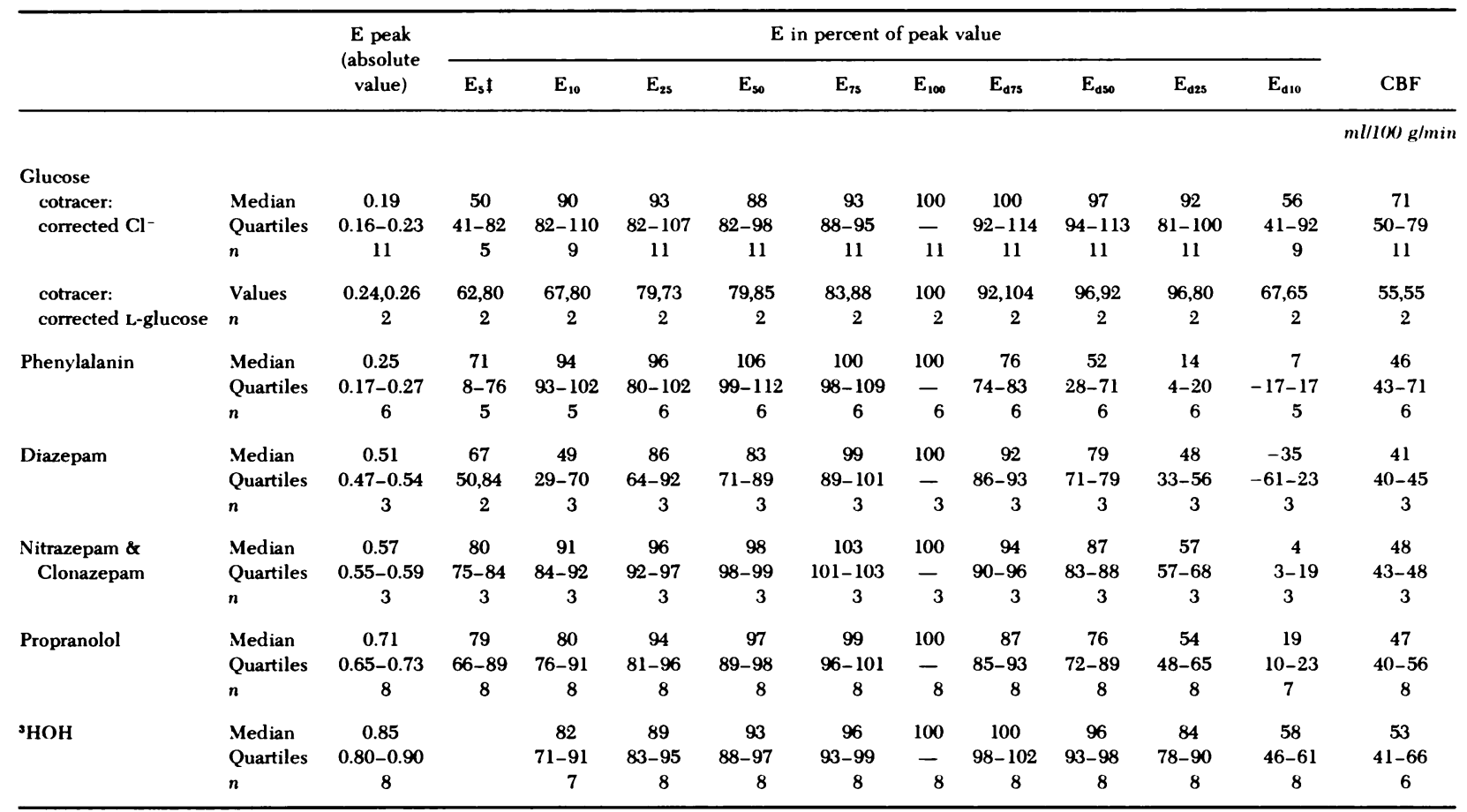

* The suffix to $\mathrm{E}$ indicates the percent saturation or desaturation, respectively, of the cotracer curve, 100 being the peak of the curve, e.g., $E_{25}$ means the $E$ at a point corresponding to $25 \%$ saturation of the cotracer on the upslope of the dilution curve, and $E_{d} 25$ the corresponding value on the downslope.

therefore, can be assumed to penetrate erythrocytes easily. A correction for difference in erythrocyte carriage was, therefore, added to In-DPTA (in an analogous manner to D-glucose vs. L-glucose). To some extent they are protein bound in blood but, because an exact estimate of the degree of binding was not possible in these experiments, we did not attempt an exact Taylor correction but used In-DTPA as the reference molecule. In any case, with these highly extracted substances, a correction for Taylor diffusion would have been small. Corrected In-DTPA = In-DTPA $+1 \frac{1}{2}\left(\mathrm{Cl}^{-}-\mathrm{Na}^{+}\right)$.

Minimal $E$ of reference substances with small molecular size often occurs and is probably the result of those areas without a blood-brain barrier (choroid plexus, area postrema, median eminence, pineal body). For L-glucose, L-lysine, $\mathrm{Na}^{+}$, and $\mathrm{Cl}^{-}$, the $\mathrm{E}$ are $1.5-2.0 \%$ (personal observation). Thus, when these were used, a correction was made by adding $2 \%$ to the amount of reference in each individual sample. For In-DTPA, such loss to brain tissue is insignificant.

\section{RESULTS}

For all of the seven compounds tested, the extraction increased during the upslope of the tracer dilution curves (Table I).

The slope of the E curves was analyzed by means of linear regression. When all the data for glucose vs. $\mathrm{Cl}^{-}$was considered, the increase was not significant although, when one experiment with a negative slope was omitted, significance was achieved $(P<0.05)$. For glucose vs. L-glucose, phenylalanine, nitrazepam- clonazepam, propranolol, and $\mathrm{HOH}$, the increase was significant $(P<0.05)$ for each group; in the case of diazepam, the individual curves also rose significantly $(P<0.05)$. The relative increase in $E$ was of the same order of magnitude for all substances studied, $E(t)$ being $\sim 90 \%$ of its maximum value at a point on the upslope of the reference curve corresponding to $25 \%$ of its peak.

Representative dilution and $\mathrm{E}$ curves for glucose and propranolol are illustrated in Figs. 4 and 5, respectively. The corrections for intravascular phenomena only have a minor influence on the shape of the $\mathrm{E}$ curves, especially with such highly extracted substances as propranolol.

In an additional group of four patients, the $\mathrm{E}$ curve for propranolol was evaluated during normal $\mathrm{CBF}$ as well as during low CBF (induced by voluntary hyperventilation) and high CBF (induced by carbon dioxide inhalation) (Table II). The flow changes had no significant influence on the shape of the $\mathrm{E}$ curve.

\section{DISCUSSION}

The increase in the $E(t)$ curve during the upslope of the tracer dilution curves cannot be explained by intravascular phenomena. This increase occurred with all the molecules tested, irrespective of transfer condi- 
TABLE II

Extraction Curve Configurations for Propranolol at Different Flow Rates

(noncorrected $\mathrm{Cl}^{-}$is the reference molecule)

\begin{tabular}{|c|c|c|c|c|c|c|c|c|c|c|c|c|}
\hline & \multirow{2}{*}{$\begin{array}{c}\begin{array}{c}\text { E peak } \\
\text { (absolute } \\
\text { value) }\end{array} \\
\text {. }\end{array}$} & \multicolumn{10}{|c|}{$\mathrm{E}$ in percent of peak value (median values) } & \multirow[b]{2}{*}{ CBF } \\
\hline & & $E_{s}$ & $E_{10}$ & $\mathrm{E}_{25}$ & $\mathrm{E}_{50}$ & $E_{75}$ & $E_{100}$ & $E_{d 75}$ & $\mathrm{E}_{\mathrm{dso}}$ & $E_{\mathrm{d} 25}$ & $E_{d 10}$ & \\
\hline & & & & & & & & & & & & $\mathrm{mll} / 100 \mathrm{~g} / \mathrm{min}$ \\
\hline \multicolumn{13}{|c|}{2 patients studied at } \\
\hline Normocapnia & 0.57 & 90 & 92 & 96 & 98 & 99 & 100 & 95 & 92 & 87 & 30 & 23,30 \\
\hline Hypocapnia & 0.60 & 85 & 88 & 95 & 96 & 98 & 100 & 95 & 93 & 79 & 44 & 20,23 \\
\hline Hypercapnia & 0.48 & 88 & 90 & 94 & 96 & 102 & 100 & 100 & 94 & 83 & 26 & 32,43 \\
\hline \multicolumn{13}{|c|}{2 patients studied at } \\
\hline Normocapnia & 0.46 & 85 & 88 & 92 & 94 & 97 & 100 & 100 & 97 & 70 & 3 & 69,80 \\
\hline Hypocapnia & 0.61 & 87 & 92 & 94 & 98 & 99 & 100 & 98 & 90 & 72 & 17 & 46,38 \\
\hline Hypercapnia & 0.36 & 76 & 90 & 96 & 98 & 99 & 100 & 103 & 94 & 65 & 0 & 107,177 \\
\hline
\end{tabular}

* The suffix to $\mathrm{E}$ indicates the percent saturation or desaturation, respectively, of the cotracer curve, 100 being the peak of the curve, e.g., $\mathrm{E}_{25}$ means $\mathrm{E}$ at a point corresponding to $25 \%$ saturation of the cotracer on the upslope of the venous outflow curve, and $E_{d 25}$ the corresponding value on the downslope.

tions. Thus, it was present for glucose and amino acids, which are transferred by specific carrier mechanisms, as well as for lipophilic substances and water, which enter the brain by dissolving in the plasma membrane phase.

A possible explanation of the increasing $\mathbf{E}$ might be that the test substances bypass the capillaries by diffusion from arteries to veins (shunting), as has been described for ${ }^{133} \mathrm{Xe}$ (14). However, this seems unlikely because the $\mathrm{E}(\mathrm{t})$ curves for $\mathrm{HOH}$ and propranolol have almost the same slope even though the free diffusion coefficient for $\mathrm{HOH}$ is much higher than that for propranolol. Furthermore, the shape of the propranolol $\mathrm{E}$ curve was not influenced by a CBF decrease, which would have been the case if shunting had been involved. The increasing $\mathrm{E}$ must, therefore, be taken as evidence for a certain degree of heterogeneity in the cerebral microcirculation. Thus, tracer particles with short transit $t$ (which correspond to capillaries with high flow rates and/or short lengths) have less contact with the blood-brain barrier and thus a lower extraction than those tracer particles with long transit t. Arteriovenous shunts - if they exist in the human brain might be included in this phenomenon.

As far as we are aware, no other data on capillary heterogeneity in the brain exist. However, such a heterogeneity is likely because there are differences betwen gray and white matter (15) and perfusion inhomogeneities within the gray matter of the cerebral cortex exist (16). In vasodilated skeletal muscle, $\mathrm{E}(\mathrm{t})$ is constant during the upslope of the tracer dilution curve, indicating that the capillaries are homogenous (17). Rose and Goresky (18) observed an increase of $\mathrm{E}(\mathrm{t})$ during the upslope of the dilution curve for sucrose in the myocardium (albumin was used as refer- ence). However, in contrast to our observations in the brain, the signs of heterogeneity disappeared in the heart at high flow because the $\mathrm{E}(\mathrm{t})$ curves flattened out. Their results suggest that capillary intermittency may occur in the vasoconstricted myocardium. It is still not known whether capillary intermittency occurs in brain, but our finding of cerebral capillary perfusion heterogeneity seems to be unrelated to capillary intermittency, the heterogeneity being of a more static nature, uninfluenced by marked $\mathrm{CBF}$ changes. It is more likely to be the result of differences in capillary length.

In the following, the implications for the calculation of the average $\mathrm{E}$ will be discussed. The presence of cerebral capillary heterogeneity and the differences in intravascular behavior of the compounds will influence the calculation of the average $\mathrm{E}$, which is the mean of the weighted values and corresponds to integration of Eq. 1. The difference in intravascular properties can be minimized by choosing test and reference substances with intravascular properties as close to each other as possible. Valid corrections for the remaining small intravascular differences can be made by integrating appropriate parts of the dilution curves as described in previous publications $(2-4,6)$. For substances with low extractions and low backdiffusion, this integration will comprise the upslope and most of the downslope of the curves, e.g., until $40 \%$ desaturation for substances with different interlaminar diffusion and until infinity for substances with different erythrocyte transport. When a large part of the curve is integrated in this way, the calculated average $E$ will be a good estimate of the true average E. However, for substances with high $\mathrm{E}$ and backdiffusion, the tail part of the outflow curve can be heavily influenced 
by the backdiffusion and cannot be used for the calculations because the calculated average $\mathrm{E}$ would be an underestimation of the true value. Thus, the increase in $\mathrm{E}(\mathrm{t})$ as the result of heterogeneity, together with the effect of backdiffusion, could lead to an underestimation of the average $E$ of substances with a high $E$. In these cases, the best solution is to calculate the average E from a short part of the outflow curves around the peak, and not from the whole upslope, because that would result in a slightly (and spuriously) lower value. Furthermore, the influence of interlaminar diffusion and erythrocyte carriage is also less pronounced around the peak of the venous outflow curves.

In conclusion, our results indicate the presence of capillary perfusion heterogeneity in the human brain. In studies using the indicator dilution method, valid estimates of the average $E$ of test substances can still be obtained if the appropriate part of the venous outflow curves is integrated.

\section{ACKNOWLEDGMENT}

This study was supported by the Danish Medical Research Council.

\section{REFERENCES}

1. Crone, C. 1963. The permeability of capillaries in various organs as determined by use of the 'indicator diffusion' method. Acta Physiol. Scand. 58: 292-305.

2. Lassen, N. A., J. Trap-Jensen, S. C. Alexander, J. Olesen, and O. B. Paulson. 1971. Blood-brain barrier studies in man using the double indicator method. Am. J. Physiol. 220: $1627-1633$.

3. Bolwig, T. G., M. M. Hertz, O. B. Paulson, H. Spotoft, and O. J. Rafaelsen. 1977. The permeability of the bloodbrain barrier during electrically induced seizures in man. Eur. J. Clin. Invest. 7: 87-93.

4. Paulson, O. B., M. M. Hertz, T. G. Bolwig, and N. A Lassen. 1977. Filtration and diffusion of water across the blood-brain barrier in man. Microvasc. Res. 13: 113-124.

5. Olesen, J., O. B. Paulson, and N. A. Lassen. 1971. Regional cerebral blood flow in man determined by the initial slope of the clearance of intraarterially injected Xenon133. Stroke. 2: 519-540.

6. Paulson, O. B., and M. M. Hertz. 1978. Blood-brain barrier permeability during shortlasting intravascular hyperosmolality. Eur. J. Clin. Invest. 8: 391-396.

7. Taylor, G. 1953. The dispersion of soluble matter in solvent flowing slowly through a tube. Proc. R. Soc. Lond. B. Biol. Sci. Series B. 219: 186-203.

8. Lassen, N. A., and C. Crone. 1970. The extraction fraction of a capillary bed to hydrophilic molecules: theoretical consideration regarding the single injection technique with a discussion of the role of diffusion between laminar streams (Taylor's effect). In Capillary Permeability. Alfred Benzon Symposium. C. Crone and N. A. Lassen, editors. Munksgaard, International Booksellers \& Publishers Ltd., Copenhagen, Denmark. 1: 48-59.

9. Larsen, O. A., and N. A. Lassen. 1964. Cerebral hematocrit in normal man. J. Appl. Physiol. 19: 571-574.

10. Tostesen, D. C. 1959. Halide transport in red blood vessels. Acta Physiol. Scand. 46: 10-41.

11. Altman, P. L., and D. S. Dittmer. 1971. Blood and other body fluids. Federation of American Societies for Experimental Biology. Bethesda, Md. 21-22.

12. Perl, W. 1975. Red cell permeability on the mean transit time of an indicator transported through an organ by red cells and plasma. Circ. Res. 36: 352-357.

13. Goresky, C. A., G. G. Back, and B. E. Nadeau. 1975. Red cell carriage of label. Circ. Res. 36: 328-357.

14. Brodersen, P., P. Sejrsen, and N. A. Lassen. 1973. Diffusion bypass of Xenon in brain circulation. Circ. Res. 32: 363-369.

15. Cobb, S. 1932. The cerebrospinal blood vessels. In Cytology and Cellular Pathology of the Nervous System. W. Penfield, editor. Paul Hoeber, Inc., New York. 575610.

16. Larsen, B., E. Skinhøj, and N. A. Lassen. 1978. Variation in regional cortical blood flow in the right and left hemispheres during automatic speech. Brain. 101: 193209.

17. Lassen, N. A., and J. Trap-Jensen. 1970. The validity of the indicator diffusion method for measuring the capillary diffusion capacity ${ }^{51} \mathrm{Cr}$-EDTA in hyperemic skeletal muscle. Eur. J. Clin. Invest. 1: 118-123.

18. Rose, C. P., and C. A. Goresky. 1976. Vasomotor control of capillary transit time heterogeneity in canine coronary circulation. Circ. Res. 39: 541-554. 